\title{
Analysis of Time-Resolved Laser Plasma Ablation using an Imaging Spectra Technique
}

\author{
H. Luna ${ }^{\#}$, J. Dardis ${ }^{\ddagger}$, D. Doria ${ }^{\dagger}$, and J.T. Costello \\ \#Centro Brasileiro de Pesquisas Físicas, Rua Xavier Sigaud 150, Urca, Rio de Janeiro, Brazil. \\ $\dagger$ Queens University Belfast, Belfast, UK. and \\ $\$ N C P S T$ and School of Physical Science, Dublin City University, Glasnevin, Dublin 9, Republic of Ireland.
}

\section{Received on 10 October, 2007}

\begin{abstract}
Pulsed laser deposition (PLD) is extensively employed for the growth of thin films. The laser-material interaction involves complex processes of heating, melting, vaporization, ejection of atoms, ions and molecules, shock waves, plasma initiation, expansion and deposition onto a substrate. The understanding of the spatial and temporal distribution of a plasma parameters in a laser-produced plasma is important to the control of thin film growth process. In this work we have studied the dynamics of laser ablated graphitic carbon plasma expanding into vacuum using a spectroscopic imaging suitable as an in situ \& automated diagnostic sampling technique for PLD. Time-resolved spectra, that were also spatially resolved in one dimension along the axis of plasma expansion, were obtained using a time-gated intensified charge-coupled device (ICCD) coupled to a stigmatic Czerny-Turner spectrograph. Plasma parameters such as electron density, temperature and plume velocity expansion were extracted directly from the analysis of the C II $\left(2 s^{2} 3 d-2 s^{2} 4 f\right)$ transition.
\end{abstract}

Keywords: Spectroscopy; Laser produced plasma; Time-resolved imaging; Plasma density; Plasma temperature

\section{INTRODUCTION}

Diamond-like carbon (DLC) materials are assuming a growing interest in a number of fields ranging from electrical and optical applications to biomedical engineering. For example, the possibility to coat large surfaces with a diamond-like carbon films makes them ideal for radiation detection, X-ray windows, biocompatible membranes, wave sensors and high voltage (HV) switching devices [1,2].

Pulsed Laser Deposition (PLD) is one of the techniques used to produce films with structures varing from a-C films (amorphous carbon films) to nanophase diamond, where the film characteristics output such as hardness, density and friction coeficient are dependent on the deposition conditions [3]. Its main advantage over other techniques is the possibility to control properties of the deposited film by changing the laser irradiation conditions such as laser beam wavelength, pulse duration, energy and focusing spot size. The carbon plasma is formed when a laser pulse with intensity of the order of $10^{9-11}$ $\mathrm{W} / \mathrm{cm}^{2}$ is focused onto a graphitic carbon target. The plasma then expands freely into vacuum or in an inert gas atmosphere. The particles ejected from plasma are subsequently deposited on different substrates to form the films.

The monitor and control of the PLD-generated plasma can be done by several diagnostic sampling techniques. Diagnostic sampling techniques may be divided into three categories: (1) those involving sample extraction and ex situ analysis, e.g. mass spectrometry; (2) those which require placing a physical probe (e.g. Langmuir probe) in the region of interest [4]; and (3) in situ optical probes, e.g. optical emission spectroscopy (OES), Thomson scattering and Raman spectroscopy.

The aim of this work is to develop a tool for in situ PLD characterization based on OES technique. For this propose we apply a space and time resolved, spectral imaging technique [5] where plasma parameters can be extracted directly from line emission in the VIS/NIR (range of interest for a table top apparatus). We have found the C II $\left(2 s^{2} 3 d-2 s^{2} 4 f\right)$ transition, to be the best choice of line emission for a rapid and ready optical characterization of the plasma during film deposition. A MATLAB ${ }^{\mathrm{TM}}$ [6] code was used for plasma parameter extraction from the image spectrum and can be adapted for on-line monitoring deposition conditions.

\section{EXPERIMENTAL APPARATUS}

The experimental apparatus is shown schematically in Fig. 1. A Q-switched Nd:YAG laser $(E=150 \mathrm{~mJ}, \lambda=1064$ $\mathrm{nm}$, pulse duration of $15 \mathrm{~ns}$ FWHM and $10 \mathrm{~Hz}$ repetition rate) is focused onto a graphitic carbon target surface placed inside a vacuum chamber $\left(5 \times 10^{-5}\right.$ mbar $)$ generating a plasma that expands normal to the target surface. A typical laser irradiance can be estimated from the crater diameter shown in Fig. 2, obtained with a SEM. In this case the shape of the spot is approximately an ellipsoid of $a=59 \mu \mathrm{m}$ and $b=78.6 \mu \mathrm{m}$ ), resulting in a laser irradiance of $\approx 7 \times 10^{10} \mathrm{~W} / \mathrm{cm}^{2}$.

The target holder was mounted on a $X Y Z$ stage in order to allow us to move to a fresh surface after exposure. The optical system used to focus the plasma onto the entrance slit of the spectrograph comprises a Dove prism located between two achromatic lenses, L2 and L3, with focal lengths of 250 $\mathrm{mm}$, respectively. The Dove prism is used to rotate the image of the plasma plume by $90^{\circ}$, projecting the direction of the plasma expansion ( $z$-axis) along the entrance slit ( $y$-axis) of a $0.5 \mathrm{~m}$ focal length Czerny-Turner spectrograph. The spectrograph was equipped with a grating of groove density of 1200 lines/mm and a blaze wavelength of $400 \mathrm{~nm}$. The optimum resolution was obtained at a slit width of $60 \mu \mathrm{m}$ since no improvement in resolution was obtained at narrower slit widths while the resolution was degraded at larger slit widths. The dispersion of the grating was found to be $0.04 \mathrm{~nm} / \mathrm{pixel}$ for $\lambda$ $=426.7 \mathrm{~nm}$ and instrument function of the system to be 0.16 $\mathrm{nm}$, measured independently.

The magnification of the optical system was found to be 1:1.5. Spectral image readout was provided by an intensi- 
Spectrograph - Czerny-Turner

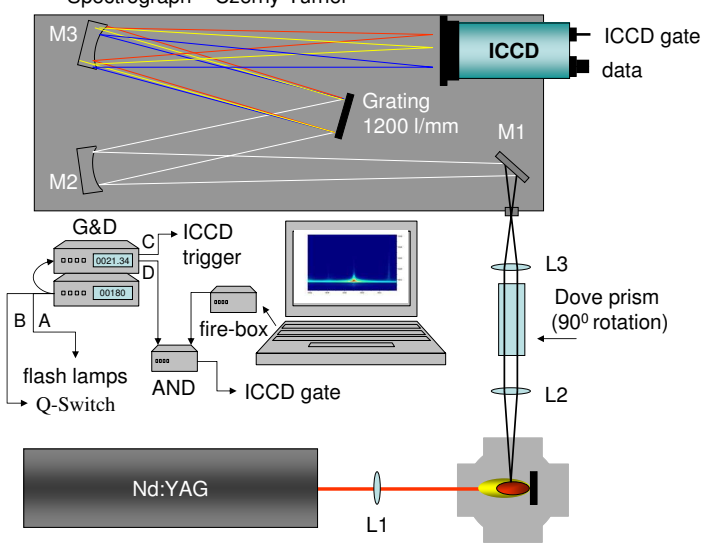

FIG. 1: Experimental set-up for the colliding plasma experiment. C target, lenses $\left(l_{1}, l_{2}\right.$ and $\left.l_{3}\right)$, wedge prism (WP), Dove prism, spectrograph and ICCD camera.

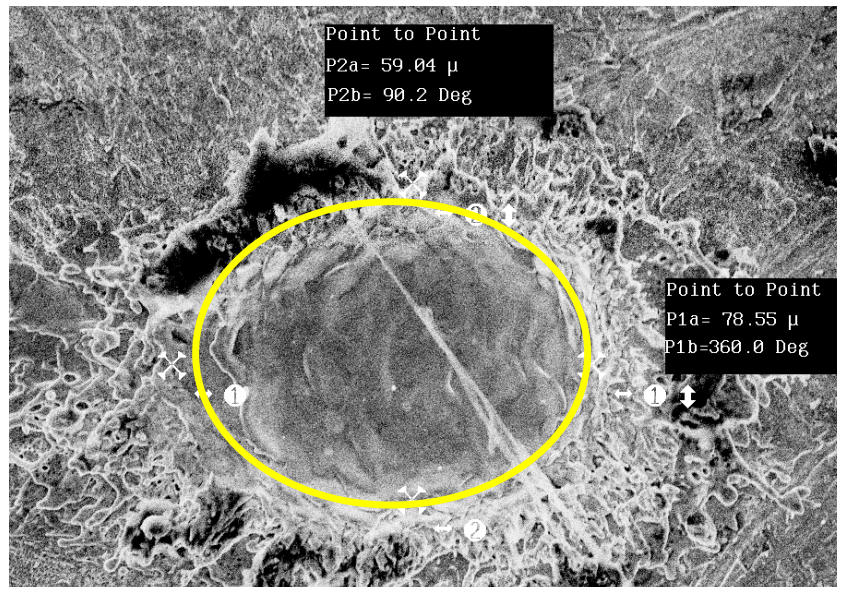

FIG. 2: Image of the target after being exposed to the laser shot. Typical spot profile obtained after laser exposure.

fied two-dimensional (2D) charge-coupled device (Andor ${ }^{\mathrm{TM}}$ ICCD). The ICCD consisted of an $18 \mathrm{~mm}$ diameter micro channel plate (MCP) image intensifier coupled to a $512 \times$ 512 pixels charge-coupled device by a high quality relay lens system. Since the spectrometer is stigmatic, the overall ICCD/spectrometer system provides 1D (spatial) by 1D (spectral) images of the plasma plume. The ICCD camera used in the experiment can be operated with a gate width as low as 2 ns FWHM. By varying the delay between plasma initiation and the opening of the ICCDs gate, we were able to track the temporal evolution of the plasma. Two Stanford DG535 ${ }^{\text {TM }}$ delay generators were used to achieve this synchronization. A 16 ns gate width was used to record the spectra used during this work.

\section{PLASMA PARAMETERS ANALYSIS}

A set of raw images (background subtracted) obtained from the plasma imaging system comprising the spectrograph (with ICCD readout) are shown in Fig. 3 for the C II $\left(2 s^{2} 3 d-2 s^{2} 4 f\right)$ transition at three different times during the plume expansion, respectively, 60, 100 and $180 \mathrm{~ns}$. The $\mathrm{x}$-axis corresponds to the direction of wavelength dispersion (spectrometer setting centred at the $426.7 \mathrm{~nm}$ transition wavelength) and the z-axis corresponds to spatial distribution of the corresponding excited state species distribution along the plasma plume expansion direction. The analyses of the spectral images are described in reference [5]. In brief, a set of cells or grid can be superimposed onto the image and the image segment enclosed within each cell binned vertically. The binned lineouts (or spectra) are then projected along the expansion direction (z-axis) and a Voigt function is used to fit each spectrum for each spatial cell or box. Therefore, spectrally resolved parameters such as relative line intensity, Gaussian and Lorentzian widths can then be extracted for each position, from the timeresolved images.

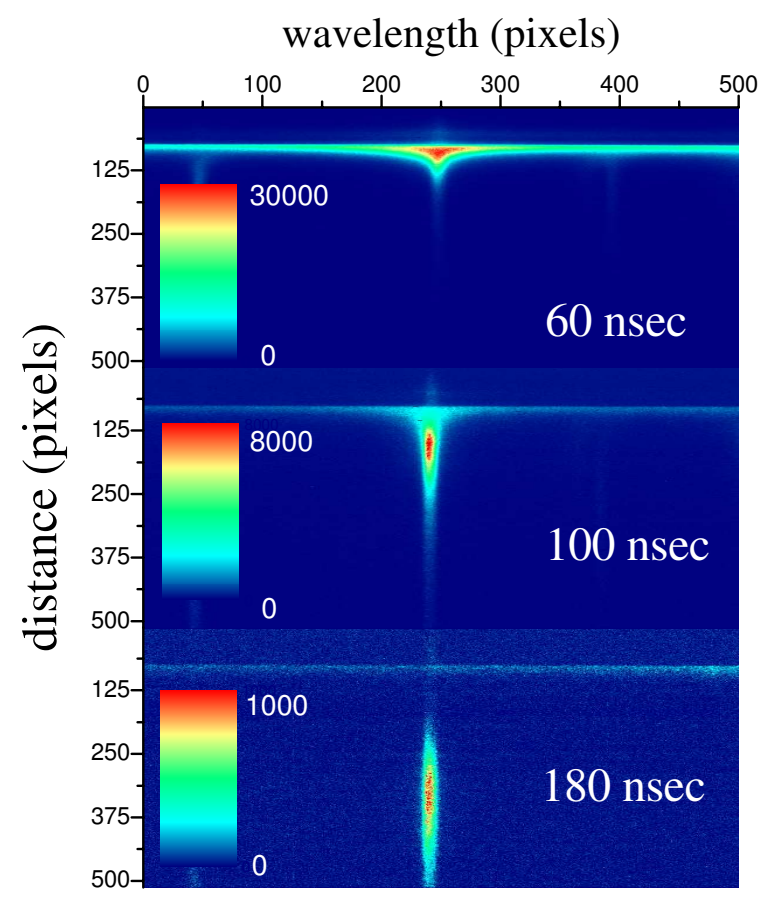

FIG. 3: Typical raw spectroscopic image (wavelength on $\mathrm{x}$-axis and longitudinal distance on y-axis) of plume expansion for the CII 426.7 $\mathrm{nm}$ transition. The spectra were recorded at a time delays of 60,100 and $180 \mathrm{~ns}$ after plasma initiation. 


\section{A. Plume expansion velocity}

The first parameter is the plume velocity and can be extracted from the spatial line intensity distribution. By tracking the maximum brightness displacement, shown in Fig. 4 for the CII $\left(2 s^{2} 3 d-2 s^{2} 4 f\right)$ transition, a velocity expansion of 0.75 $\times 10^{7} \mathrm{~cm} / \mathrm{sec}$ was obtained, towards the longitudinal direction, for the singly ionized component of the plume. This is in good agreement with the value measured by Harilal and co-workers $\left(10^{7} \mathrm{~cm} / \mathrm{sec}\right)$, using a 2-D fast photography technique for a carbon plasma expanding in vacuum [7].

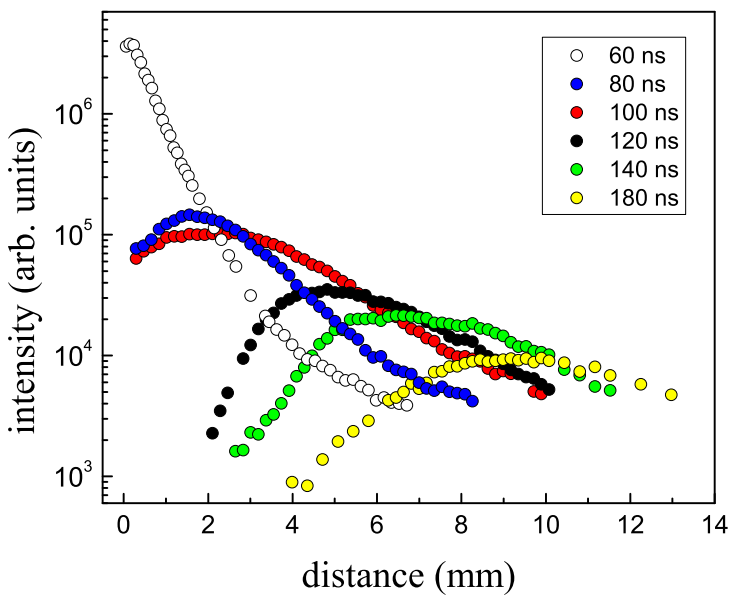

FIG. 4: Longitudinal line intensity distribution for the CII $426.7 \mathrm{~nm}$ transition.

Concerning the theoretical treatment of the plume expasion, two models are often used for comparison with the experimental results. Special solutions of gas dynamics equations can be obtained from an arbitrary initial temperature profile and laser irradiance conditions. The simplest case assume an isothermal solution of the gas-dinamical equations with gaussian pressure and density profiles [8]. However spatially and time resolved experiements [5] have shown that the spatial distributions of the electron temperature are not isothermal and disregarding spatial temperature gradients is physically inadequate. A more realistic model is presented by Anisimov and co-workes $[9,10]$. In their work a model for the plume expansion is obtained from the analitical solution of the gasdinamical equations considering a non-isothermal adiabatic expansion of the plume in vacuum or a gas atmosphere. A gas dynamics expansion code based on this model is currently under development by us and results from it will be presented later.

\section{B. Electron density}

The second parameter to be extracted is the electron density. Under the assumption of LTE (local thermodynamics equilibrium), the electron density can be calculated by analysing the profile of the C II $\left(2 \mathrm{~s}^{2} 3 \mathrm{~d}-2 \mathrm{~s}^{2} 4 \mathrm{f}\right)$ transition centered at $\lambda_{0}$ $=426.7 \mathrm{~nm}$. A Voigt function is used to fit a profile to the transition that takes into account the various processes that contribute to the line broadening. Several broadening mechanism can be attributed to the Lorentzian and Gaussian component. The Stark contribution to the broadening is described by the Lorentzian component and all other contributions are accounted for the Gaussian components. Two broadening mechanism are often important for laser produced plasma [11, 12]. The broadeing component due to Doppler effect, can be calculated from: $\lambda_{1 / 2}(\mathrm{FHWM})=3.8 \times 10^{-5} \lambda_{0}(k T / M)^{1 / 2}$, where $k T$ is the plasma temperature in $\mathrm{eV}, M$ is the atomic mass number and $\lambda_{0}$ is the line wavelength in $\mathrm{nm}$, and the instrument function of the apparatus which is a function of the optics, spectrograph and ICCD configuration. For the transition studied in this work we have found the Doppler contribution to be $\approx 0.005-0.015 \mathrm{~nm}$, for $\mathrm{T}=1-10 \mathrm{eV}$. The second contribution comes from the instrument function which was measured independently using the scattered light from a HeNe laser and is well approximated by a Gaussian profile with a full width at half maximum of $0.16 \mathrm{~nm}$.

Therefore, having obtained the pure Stark component, the electron density can be obtained by solving the equations below:

$$
\Delta \lambda_{\text {width }}=2 W\left(N_{e} / 10^{16}\right)+3.5 A\left(N e / 10^{16}\right)^{1 / 4}
$$

$$
\times\left[1-1.2 N_{D}^{-1 / 3}\right] W\left(N_{e} / 10^{16}\right)
$$

where the electron impact parameter $W$, and the ionic-impact parameter $A$ are taken from tabulated values $[12,17]$ and $N_{D}$ is the number of particles in the Debye sphere, given by:

$$
N_{D}=1.72 \times 10^{9}\left(T^{3} / N_{e}\right)^{1 / 2}
$$

The first term in equation 1 describes the broadening caused by electron-emitter collisions. The second term, or quasistatic component describes the broadening due to ion-emitter interactions. Stark broadening in lowly ionised species is dominated by electron collisions, thereby allowing us neglect the ion broadening term and to use only the first term in the equation 1 . The FHWM $\left(\Delta \lambda_{\text {width }}\right)$ will then scale linearly with the electron density $N_{e}$.

The spatial distributions of the electron density are shown in Fig. 5 for three plasma expansion times, 60, 100 and 180ns after plasma initiation. It should be noted that there is a sharp decrease in the magnitude of the electron density for distances near to the target surface (near $0.5 \mathrm{~mm}$ ), as shown for 60,80 and 100ns (top graph). For later observation times (180 ns) the emission intensity begins to fade (see Fig. 4) and the electron density distribution decreases by almost two orders of magnitude, becoming reasonably flat along the expansion direction (longitudinal). 


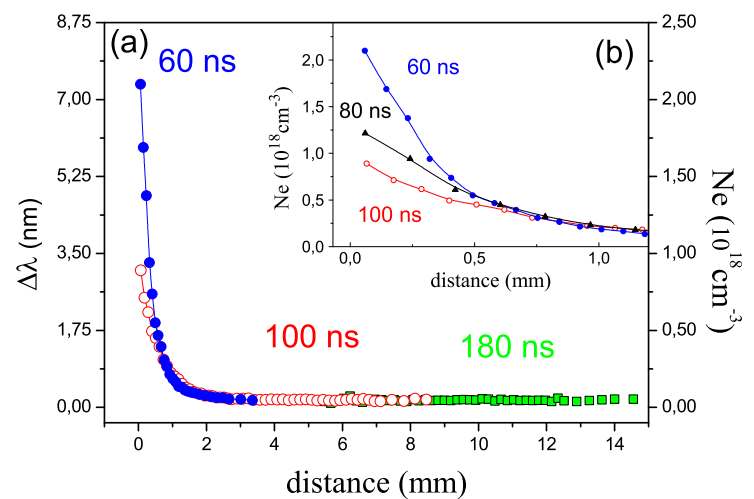

FIG. 5: (a) Spatial distribution of the electron density for 60, 100 and $180 \mathrm{~ns}$. On (b) Insert- expanded view of the region near the target surface showing the sharp decay in the magnitude of $N_{e}$.

\section{Electron temperature}

Finally, the third parameter of interest to PLD, is the plasma temperature. Also, under the assumption of LTE, the electron temperature $T_{e}$ can be assumed equal to the excitation temperature $T_{\text {exc }}$, and the plasma temperature $T$ can be determined from optical emission by three methods, namely, the relative line-to-continuum intensity ratio the relative, the relative lineto-line intensity ratio for transitions from the same element and same ionization stage (Boltzmann plot), or by two subsequent ionization stages of the same element (Saha equation) $[11,12]$. Once the main goal of this work, is to present an in - situ \& automated plasma characterization procedure for PLD, any temperature calculation based on line-to-line ratio becomes only achievable if two or more line transitions lie within the spectral window of the spectrograph. For a CzernyTurner specrograph, the choice of the diffraction grating will determine the spectral range and resolution. For example, a grating with density of 300 lines/mm can provide a larger spectral range (typically $300-700 \mathrm{~nm}$ or $500-1100 \mathrm{~nm}$ depending on the blaze angle), allowing simultaneous measurement of several lines and the use of Boltzmann plot method. Larger spectral range comes with the expense of losing spectral resolution (typically 0.5 to $1 \mathrm{~nm}$ ) and consequently the impossibility of measuring densities via Stark broadening. Higher density gratings (1200 to 3600 lines/mm) are suitable for Stark measuremnts but the spectral range window will not be wide enough to allocate two or more emission line transition from the same element. In this work, as only one line is available within the spectral window of the spectrometer plus CCD spectral window, we set on the relative line-to-continuum intensity ratio method to to measure the electron temperature.

The background continuum from a plasma arises from the interactions of free electrons with ions yielding recombinative radiation (free-bound transitions) and Bremsstrahlung emission due to freefree transitions, and can be described by the following equation $[13,14]$,
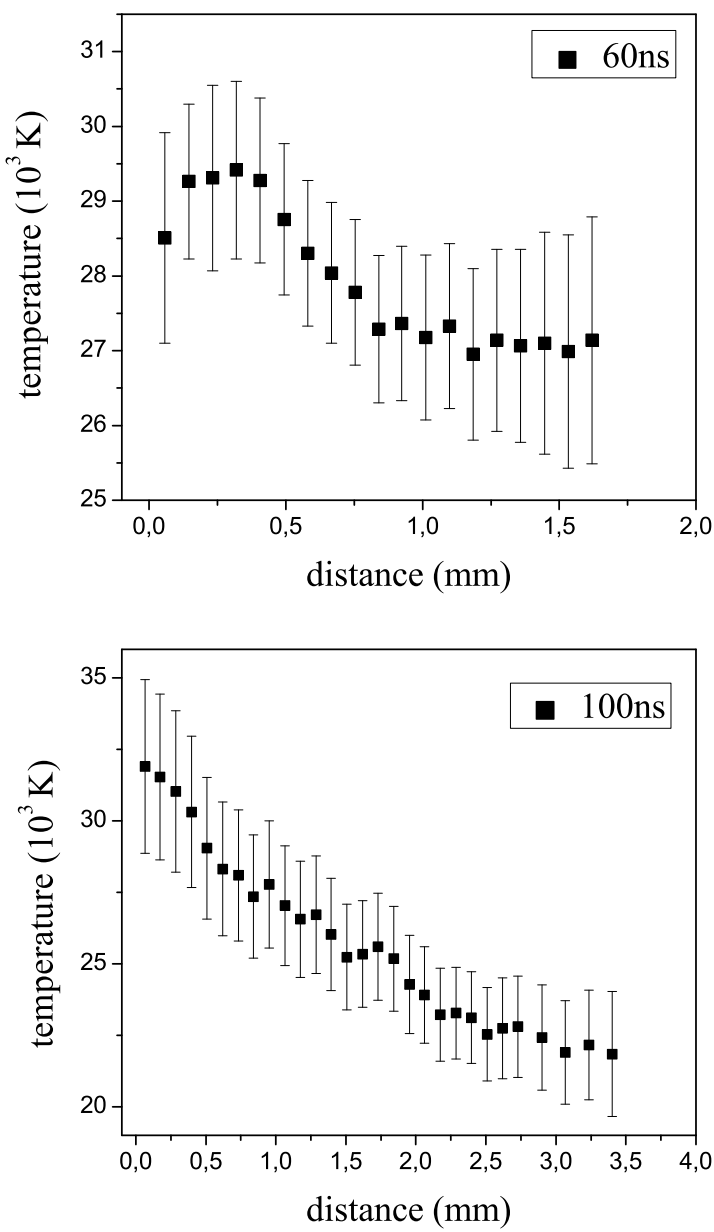

FIG. 6: Typical electron temperatures obtained using the line-tocontinuum ratio method.

$$
\varepsilon_{c}=C \frac{N_{e}^{2}}{\lambda^{2} T_{e}^{1 / 2}}\left\{\xi\left[1-\exp \left(\frac{-h c}{\lambda k T_{e}}\right)\right]+G\left[\exp \left(\frac{-h c}{\lambda k T_{e}}\right)\right]\right\}
$$

where $\mathrm{C}=1.632 \times 10^{-43}\left[\mathrm{Jm}^{4} \mathrm{~K}^{1 / 2} \mathrm{sec}^{-1} \mathrm{sr}^{-1}\right]$ and $\lambda, N_{e}$, and $T_{e}$ are, respectively, wavelength, electron density and temperature. The constants $h, c$ and $k$ are the Plancks constant, the speed of light and Boltzmanns constant. The free-bound and free-free factors are denoted by $\xi$ and $G$, respectively. From the Saha equation, the equation for the relative line-tocontinuum intensity ratio is given by:

$$
\frac{\varepsilon_{i}}{\varepsilon_{c}}=\frac{C_{r} A_{k i} g_{k}}{Z_{i}} \frac{\lambda}{T_{e}} \frac{\exp \left(\Delta E / k T_{e}\right)}{\left\{\xi\left[1-\exp \left(\frac{-h c}{\lambda k T_{e}}\right)\right]+G\left[\exp \left(\frac{-h c}{\lambda k T_{e}}\right)\right]\right\}}
$$

where, $C_{r}=2 \times 10^{-5}[\mathrm{sK}]$, and $A_{k i} g_{k}$ is the Einstein transition probability weighted by the upper energy level degeneracy. $\Delta E=E_{i}-E_{k}$, where $E_{i}$ and $E_{k}$ are the ionization potential 
and the upper level energy, respectively. For carbon we have used $\xi=1.4$ obtained for Si in references $[15,16]$ and $G=1$.

It is important to note that, equation 4 is only applicable for early stages of the plume expansion, since after $100 \mathrm{nsec}$ the continuum emission has disappeared. Secondly, it is not ideal for predicting spatial temperature profiles, because it fails to give an accurate line-to-continuum ratio at the edges of the plume, where the continuum signal is weak. In the early phase of the plume expansion (and near the target surface) the plasma emission tends to be continuum dominated and the measurement of the transition emission becomes imprecise. At the end of the plume life, the opposite scenario takes over, and the continuum emission becomes much lower than discrete line emission. Equation 4 can be solved numerically or graphically. The results are shown in Fig. 6, for two times in the plume expansion, where the continuum emission was still measurable.

\section{CONCLUSION}

In this work we have presented an optical imaging method capable to provide space and time resolved spectra of a plasma plume during pulsed laser deposition. From a single line emission, plasma parameters such as plume expasion velocity, electron temperature and density, can be obtained in-situ and simultaneously with the film deposition. These time resolved spectra and the parameters obtained from them can be used as a fingerprint of the film deposited and as a historical record of the conditions in which the films were growth.

\section{Acknowledgments}

We thank Dr. D.F. Franceschini for helpful comments and suggestions. This work was supported by Science Foundation Ireland under the Frontiers Programme. DD acknowledges suport from EU FP6 Marie Curie Training Site Scheme. HL acknowledges support from the EU FP6 Marie Curie IIF and HEA North-South programmes.
[1] C.E. Nebel, D. Shin, B. Rezek, N. Tokuda, H. Uetsuka, and H. Watanabe J. R. Soc. Interface, 4, 439 (2007).

[2] M. Nesladek Semiconductor Science and Technology, 20, R19 (2005).

[3] A.A. Voevodin, M.S. Donley Surface and Coatings Technology, 82, 199 (1996).

[4] James G. Lunney, Brendan Doggett, and Yitzhak Kaufman, J. Phys.: Conf. Ser. 59, 470 (2007).

[5] D. Doria. K.D. Kavanagh, J.T. Costello, and H. Luna Meas. Sci. Technol. 17, 670 (2006).

[6] K.D. Kavanagh, PhD Thesis, Dublin City University (2006).

[7] S.S. Harilal, B. O'Shay, M.S. Tillack, C.V. Bindhu, and F. Najmabadi Ieee Transactions on Plasma Science, 33, (2005).

[8] R.K. Singh and J. Narayan Phys. Rev. B 41, 8843 (1990).

[9] S.I. Anisimov, D. Bauerle, and B.S. Lurkyanchuk Phys. Rev. B 48, 12076 (1993).

[10] S.I. Anisimov, B.S. Luk'yanchuk, and A. Luches Applied Sur- face Science 96-98, 24 (1996).

[11] H. R. Griem 1964 Plasma Spectroscopy (McGraw-Hill, New York).

[12] H. R. Griem 1974 Spectral Line Broadening by Plasmas (Academic Press, New York).

[13] G.J. Bastiaans and R.A. Mangold Spectrochimica Acta Part B 40B, 885 (1985).

[14] G.C.Y. Chan and W-T Chan Spectrochimica Acta Part B 57, $1771(2002)$

[15] H.C. Liu, X.L. Mao, J.H. Yoo, and R.E. Russo Spectrochimica Acta Part B 54, 1607 (1999).

[16] X. Zeng, S.S. Mao, H.C. Liu, X.L. Mao, R. Greif, and R.E. Russo Spectrochimica Acta Part B 58, 867 (2003).

[17] C. Pérez, I. de la Rosa, A.M. de Frutos, and S. Mar Phys. Rev. A 44, 6948 (1991). 